\title{
PReS-FINAL-2194: Evidence-based clinical classification criteria for periodic fevers
}

\author{
S Federici ${ }^{1 *}$, S Ozen ${ }^{2}$, I Koné-Paut ${ }^{3}$, H Lachmann ${ }^{4}$, P Woo ${ }^{5}$, L Cantarini ${ }^{6}$, G Amaryan ${ }^{7}$, A Insalaco ${ }^{8}$,

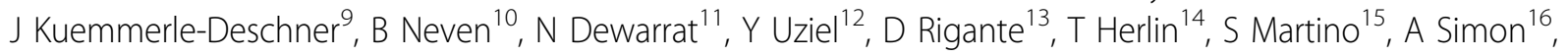 \\ S Stojanov ${ }^{17}$, H Ozdogan $^{18}$, J Frenkel ${ }^{19}$, N Ruperto ${ }^{1}$, A Martini $^{1}$, MP Sormani ${ }^{1}$, M Hofer $^{20}$, M Gattorno $^{1}$ \\ From 20th Pediatric Rheumatology European Society (PReS) Congress \\ Ljubljana, Slovenia. 25-29 September 2013
}

\section{Introduction}

No evidence-based classification criteria are so far available for the majority of autoinflammatory diseases

\section{Objectives}

To elaborate and validate a set of clinical criteria able to correctly classify patients affected with the most common periodic fevers

\section{Methods}

All FMF, TRAPS, MKD and CAPS patients enrolled in the Eurofever registry until March 2013 were evaluated. For each disease gold standards were considered according to the following criteria: i) clinical validation by centers and disease-principal investigator, ii) confirmative molecular analysis (2 mutations for MEFV with at least one mutation in exon 10, 2 mutations of MVK gene, 1 mutation of TNFRFS1A with exclusion of low-penetrance variants, 1 mutation of NLRP3 with exclusion of low-penetrance variants), iii) PFAPA patients validated by disease-principal investigator and confirmed by the centers on the basis of the follow-up. Clinical criteria were formulated on the basis of a univariate and multivariate analysis in a first group of patients (training set) and then validated in an independent set of patients (validation set).

\section{Results}

A total of 1204 consecutive patients with periodic fevers were enrolled in the registry. Among them 743 consecutive gold standard patients (288 FMF, 73 MKD, 96 TRAPS, 87 CAPS, 199 PFAPA) were evaluated (440 in

${ }^{1}$ 2nd Division of Pediatric, Gaslini Institute, Genoa, Italy

Full list of author information is available at the end of the article the training set and 303 in the validation set). The multivariate analysis identified the clinical variables (either as presence or absence) independently correlated to for each disease with their specific weight. The cut off value of the classification score was chosen on the ROC curve in order to guarantee the highest sensitivity and specificity.

The classification score was than tested in an independent set of patients (validation set) revealing a sensitivity of $93 \%$ and specificity of $89 \%$ for FMF; a sensitivity of $100 \%$ and specificity of $74 \%$ for TRAPS; a sensitivity of $80 \%$ and specificity of $90 \%$ for MKD and sensitivity of $97 \%$ and specificity of $92 \%$ for CAPS; sensitivity of $99 \%$ and specificity of $96 \%$ for PFAPA. The performance in non-gold standard patients (i.e. heterozygous patients in autosomal recessive diseases or patients with lowpenetrance mutations) revealed a variable percentage of patients (70\% FMF, 75\% TRAPS, 41\% MKD and 94\% CAPS) positive for the respective criteria.

\section{Conclusion}

Evidence-based clinical criteria for the classification of patients with inherited periodic fevers have been elaborated. These clinical criteria could be used in association with molecular analysis and other variables (i.e. metabolic examinations, response to specific treatments) for patients classification.

\section{Disclosure of interest}

None declared.

\section{Authors' details \\ 2nd Division of Pediatric, Gaslini Institute, Genoa, Italy. ${ }^{2}$ Department of Pediatric Nephrology\&Rheumatology, Hacettepe University, Faculty of Medicine, Ankara, Turkey. ${ }^{3}$ Division of Pediatric Rheumatology, Bicêtre}


Hospital, Paris, France. ${ }^{4}$ University College Medical School, London, UK. ${ }^{5}$ UCL, London, UK. ${ }^{6}$ Rheumatology Unit, University of Siena, Siena, Italy.

${ }^{7}$ Republican Children's FMF Center, Yerevan State Medical University, Yerevan, Armenia. ${ }^{8}$ Department of rheumatology, Ospedale Bambin Gesù, Rome, Italy. ${ }^{9}$ Division of Pediatric Rheumatology, University Hospital Tübingen, Tuebingen, Germany. ${ }^{10}$ Hôpital Necker-Enfants Malades, Paris, France. ${ }^{11}$ Gaslini Institute, Genoa, Italy. ${ }^{12}$ Department of Paediatrics, Meir Medical Center, Kfar Saba, Israel. ${ }^{13}$ Department of Pediatric Sciences, Università Cattolica del Sacro Cuore, Roma, Italy. ${ }^{14}$ Department of Pediatrics, Aarhus University Hospital, Aarhus, Denmark. ${ }^{15} \mathrm{Clinica}$ Pediatrica, University of Torino, Torino, Italy. ${ }^{16}$ Department of General Internal Medicine, Radboud University of Nijmegen Medical Center, Nijmegen, The Netherlands.

${ }^{17}$ National Institute of Arthritis and Musculoskeletal and Skin Diseases, $\mathrm{NIH}$, Bethesda, USA. ${ }^{18}$ Department of Internal Medicine, Istanbul University, Istanbul, Turkey. ${ }^{19}$ University Medical Center Utrecht, Utrecht, The Netherlands. ${ }^{20}$ Centre Multisite Romand de Rhumatologie Pediatrique, CHUV, Lausanne, Switzerland.

Published: 5 December 2013

doi:10.1186/1546-0096-11-S2-029

Cite this article as: Federici et al.: PReS-FINAL-2194: Evidence-based clinical classification criteria for periodic fevers. Pediatric Rheumatology 2013 11(Suppl 2):O29.

\section{Submit your next manuscript to BioMed Central and take full advantage of:}

- Convenient online submission

- Thorough peer review

- No space constraints or color figure charges

- Immediate publication on acceptance

- Inclusion in PubMed, CAS, Scopus and Google Scholar

- Research which is freely available for redistribution

Submit your manuscript at www.biomedcentral.com/submit 